\title{
Is multisystem inflammatory syndrome related with coronavirus disease 2019, Kawasaki disease, and angiotensin-converting enzyme 2 in children?
}

Lucy Youngmin Eun, MD, PhD

Department of Pediatrics, Yonsei University College of Medicine, Seoul, Korea

\section{Key message}

With the coronavirus disease (COVID-19) pandemic, it is important to consider why Kawasaki disease (KD) is prevalent in young children and its symptoms are similar to multisystem inflammatory syndrome in older children. We hypothesized that angiotensin-converting enzyme 2 (ACE2) expression may be related. In fact, this may be an answer why COVID-19 occurs less frequently in children with lower ACE2 expression.

South Korea was one of the first countries affected by coronavirus disease 2019 (COVID-19). Due to this rapidly spreading infection, South Korea adopted strict social distancing measures. The government closed schools and private institutions and encouraged people to avoid outings, observe quarantine, and wear masks in public. Correspondingly, the incidence of other viral infections in Korean children in 2020 decreased compared to those reported during the same period in previous years. During the outbreak, severe acute respiratory syndrome coronavirus 2 (SARS-CoV-2) infection was less common and less severe in children than in adults. This may be because children are less frequently exposed to its main source of transmission. ${ }^{1)}$

Currently, there are many international reports on children and adolescents diagnosed with multisystem inflammatory syndrome in children (MIS-C), a disease that shows Kawasaki disease (KD)-like clinical features. ${ }^{2-5)}$ According to the World Health Organization, MIS-C can be diagnosed in patients aged 0-19 years presenting with fever lasting for $\geq 3$ days and elevated inflammatory markers, no evidence of other microbial pathogens, and evidence of or exposure to COVID-19. Patients must also have 2 of the following features: (1) rash or bilateral nonpurulent conjunctivitis or signs of mucocutaneous inflammation; (2) hypotension or shock; (3) features of myocardial dysfunction, pericarditis, valvulitis, or coronary abnormalities; (4) evidence of coagulopathy; and (5) acute gastrointestinal (GI) problems. ${ }^{6}$

During the COVID-19 pandemic, children have been less affected than adults in South Korea. However, after the COVID-
19 outbreak, patients newly diagnosed with KD demonstrated a deterioration of biomarkers with evidence of immunocompromise and features of sepsis. This was an unusual form of $\mathrm{KD}$ or a form indicating possible KD-like MIS-C with shock. Although it is uncertain whether COVID-19 directly influences this change, it is important to pay careful attention because cases of MIS-C related to COVID-19 in children seem to be on the rise internationally. ${ }^{3-6)}$

As most MIS-C cases were presented in Europe and the United States during the pandemic, rare reports are available of MIS-C in Korea, ${ }^{7}$ implying that different genetic susceptibilities might play a role in the pathogenesis of MIS-C.

As SARS-CoV-2 infects humans through the angiotensinconverting enzyme 2 (ACE2) receptor, the ACE2 receptor might be among the key factors responsible for vascular involvement in SARS-CoV-2 infection. The affinity of SARS-CoV-2 for the ACE2 receptor may render the vascular endothelial layer susceptible to attack. Patients present with an erythematous rash and vasculitis similar to the clinical manifestations of $\mathrm{KD} .^{8-}$ 11) Many factors have been associated with both altered ACE2 expression and COVID-19 severity and progression, including age, sex, ethnicity, medication, and several comorbidities such as cardiovascular disease and metabolic syndrome. ${ }^{10,11)}$

In addition, most MIS-C patients were older than KD patients and presented with GI irritation. As previous research presented that coronavirus in the GI tract is associated with greater intestinal inflammation, the ACE2 receptor is a potential link between SARS-CoV-2 infection and intestinal inflammation. ${ }^{12}$

Furthermore, Bunyavanich et al. ${ }^{13)}$ reported that ACE2 gene expression was lower in children and higher in adults. Moreover, in younger children, ACE2 expression might develop earlier in the GI tract than in the respiratory tract.

Similarly, ACE2 is widely distributed in various human tissues, and many of its determinants have been well recognized; however, ACE2-expressing organs do not equally participate in COVID-19 pathophysiology, implying that other mechanisms are involved in coordinating cellular infection resulting in tissue

Corresponding author: Lucy Youngmin Eun, MD, PhD. Department of Pediatrics, Yonsei University College of Medicine, 50-1, Yonsei-Ro, Seodaemun-gu, Seoul 03722, Korea 
damage.

With the COVID-19 pandemic, it is important to consider why $\mathrm{KD}$ is prevalent in young children and its symptoms are similar to those of MIS-C in older children. It can be hypothesized that ACE2 expression may be related. This might be an answer to why COVID-19 occurs less frequently in children with lower ACE2 expression.

All pediatricians should be aware of all KD-like symptoms or toxic shock syndrome, even in the absence of a confirmed diagnosis of COVID-19, and MIS-C should be considered during the COVID-19 pandemic to ensure proper treatment without the misdiagnosis or overdiagnosis of $\mathrm{KD}$.

\section{Conflicts of interest}

The authors report no potential conflicts of interest relevant to this article.

See the article "Clinical features, diagnosis, and outcomes of multisystem inflammatory syndrome in children associated with coronavirus disease 2019" via https://doi.org/10.3345/cep. 2020.01900 .

\section{References}

1. Zimmermann P, Curtis N. Coronavirus infections in children including COVID-19. Ped Infec Dis J 2020;39:355-68.

2. Verdoni L, Mazza A, Gervasoni A, Martelli L, Ruggeri M, Ciuffreda M, et al. An outbreak of severe Kawasaki-like disease at the Italian epicenter of the SARS-CoV-2 epidemic: an observational cohort study. Lancet 2020; 395:1771-8.

3. European Centre for Disease Prevention and Control (ECDC). Paediatric inflammatory multisystem syndrome and SARS-CoV-2 infection in children - 15 May 2020 [Internet]. Solna (Sweden): ECDC; 2020 [cited 2020 May 31]. Available from: https://www.ecdc.europa.eu/en/ publications-data/paediatric-inflammatory-multisystem-syndrome-andsars-cov-2-rapid-risk-assessment.
4. U.S. Centers for Disease Control and Prevention (CDC). Multisystem inflammatory syndrome in children (MIS-C) associated with coronavirus disease 2019 (COVID-19) [Internet]. Bethesda (MD): CDC; 2020 May [cited 2020 May 31]. Available from: https://emergency.cdc.gov/han/ 2020/han00432.asp.

5. Riphagen S, Gomez X, Gonzalez-Martinez C, Wilkinson N, Theocharis P. Hyperinflammatory shock in children during COVID-19 pandemic. Lancet 2020;395:1607-8.

6. World Health Organization (WHO). Multisystem inflammatory syndrome in children and adolescents with COVID-19. Scientific brief [Internet]. Geneva (Switzerland): WHO; 2020 May [cited 2020 May 31]. Available from: https://www.who.int/publications-detail/multisysteminflammatory-syndrome-in-children-and-adolescents-with-covid-19.

7. Kwak JH, Lee SY, Choi JW; Korean Society of Kawasaki Disease. Clinical features, diagnosis, and outcomes of multisystem inflammatory syndrome in children associated with coronavirus disease 2019. Clin Exp Pediatr 2021;64:68-75.

8. South AM, Diz DI, Chappell MC. COVID-19, ACE2, and the cardiovascular consequences. Am J Physiol Heart Circ Physiol 2020;318: H1084-H1090.

9. Guo J, Huang Z, Lin L, Lv J. Coronavirus Disease 2019 (COVID-19) and cardiovascular disease: a viewpoint on the potential influence of angiotensin-converting enzyme inhibitors/angiotensin receptor blockers on onset and severity of severe acute respiratory syndrome coronavirus 2 infection. J Am Heart Assoc 2020;9:e016219.

10. Dalan R, Bornstein SR, El-Armouche A, Rodionov RN, Markov A, Wielockx B, et al. The ACE-2 in COVID-19: foe or friend? Horm Metab Res 2020;52:257-63.

11. Yan R, Zhang Y, Li Y, Xia L, Guo Y, Zhou Q. Structural basis for the recognition of the SARS-CoV-2 by full length human ACE-2. Science 2020;367:1444-8.

12. Garg M, Royce SG, Lubel JS. Letter: intestinal inflammation, COVID-19 and gastrointestinal ACE2-exploring RAS inhibitors. Aliment Pharmacol Ther 2020;52:569-70.

13. Bunyavanich S, Do A, Vicencio A. Nasal gene expression of angiotensinconverting enzyme 2 in children and adults. JAMA 2020;323:2427-9.

How to cite this article: Eun LY. Is multisystem inflammatory syndrome related with coronavirus disease 2019, Kawasaki disease, and angiotensin-converting enzyme 2 in children? Clin Exp Pediatr 2021;64:225-6. https://doi.org/10.3345/cep.2021. 00031. 